\title{
Sistemas de salud mental en El Salvador, Guatemala y Nicaragua. Resultados de una evaluación mediante el WHO-AIMS
}

\author{
Jorge Jacinto Rodríguez, ${ }^{1}$ Thomas Barrett, ${ }^{2}$ Silvia Narváez, ${ }^{3}$ \\ José Miguel Caldas, ${ }^{4}$ Itzhak Levav ${ }^{5}$ y Shekhar Saxena ${ }^{2}$
}

Forma de citar

Rodríguez JJ, Barrett T, Narváez S, Caldas JM, Levav I, Saxena S. Sistemas de salud mental en El Salvador, Guatemala y Nicaragua: resultados de una evaluación mediante el WHO-AIMS. Rev Panam Salud Publica. 2007;22(5):348-57.

RESUMEN Los autores realizaron una evaluación de los sistemas de salud mental en El Salvador, Guatemala y Nicaragua, por medio de un grupo de indicadores seleccionados. Para recopilar la información en los países se utilizó el Instrumento de Evaluación para Sistemas de Salud Mental de la Organización Mundial de la Salud (WHO-AIMS, por su sigla en inglés). Nicaragua, Guatemala y El Salvador tienen serias limitaciones en sus sistemas nacionales de salud mental, en especial en la atención primaria, así como una marcada insuficiencia de recursos humanos calificados. El presupuesto dedicado a la salud mental apenas representa 1\% del presupuesto general de salud y los hospitales psiquiátricos situados en la capital de los países consumen más de $90 \%$ de los fondos de salud mental. Los limitados recursos (materiales y humanos) existentes se concentran en las respectivas capitales de los países. No se han formulado politicas ni legislaciones nacionales de salud mental. Sin embargo, las tres naciones cuentan con planes nacionales en ejecución. Asimismo, se ha avanzado en el diseño e implementación de programas de protección de la salud mental en situaciones de desastre. Es necesario establecer acuerdos con las procuradurías de los derechos humanos para incrementar la vigilancia y protección de los derechos humanos en las personas afectadas por enfermedades mentales.

En los últimos años se han logrado impulsar algunas experiencias innovadoras que requieren ser generalizadas. La Organización Panamericana de la Salud (OPS/OMS) y el proyecto WHO-AIMS han contribuido al desarrollo de modelos comunitarios de servicios de salud mental. También se identificaron prioridades y se plantean recomendaciones para la acción.

Palabras clave Salud mental, servicios de salud mental, evaluación de servicios de salud, El Salvador, Guatemala, Nicaragua.

1 Organización Panamericana de la Salud, Unidad de Salud Mental, Abuso de Sustancias y Rehabilitación. La correspondencia se debe enviar a Jorge Rodríguez, Unit Chief, Mental Health, Substance Abuse \& Rehabilitation, PAHO, 525 23rd Street, NW, Washington, D.C., 20037, Estados Unidos de América. Correo electrónico: rodrigjo@paho.org

2 World Health Organization, Noncommunicable Diseases and Mental Health Cluster, Mental Health and Substance Abuse Department, Mental Health: Evidence and Research Team, Ginebra, Suiza.

3 Organización Panamericana de la Salud/Organización Mundial de la Salud, Oficina del Representante en Nicaragua, Managua, Nicaragua.
La situación de la salud mental en Centroamérica obedece a la presencia de factores complejos y la población de estos países ha quedado marcada por múltiples y numerosos eventos

\footnotetext{
Universidad de Nova Lisboa, Lisboa, Portugal.

5 Cuando se realizó el estudio, Organización Panamericana de la Salud, Unidad de Salud Mental, Abuso de Sustancias y Rehabilitación, Washington, D.C., Estados Unidos de América.
}

traumáticos, como son los desastres naturales y los sangrientos conflictos armados. Estos acontecimientos han dejado profundas heridas y cicatrices psicopatológicas y psicosociales tanto en el plano individual como en el comunitario, además de haber generado discapacidad en las áreas del funcionamiento familiar y laboral, y ocasionado importantes pérdidas eco- 
nómicas (1-3). El impacto de estos eventos se acrecienta en virtud de haberse producido, y producirse, en un contexto de marcada pobreza. Estas condiciones adversas, sumadas a la exclusión social de grupos poblacionales, en especial indígenas y comunidades rurales, perpetúan un ciclo vicioso de sufrimiento y pobreza. Los trastornos psicopatológicos, que aumentan en relación inversa con la clase socio-económica (1), obstaculizan los esfuerzos que los grupos de población más vulnerables puedan hacer para superar ese ciclo.

No obstante la situación antes descrita, en casi todos los países centroamericanos los sistemas de salud mental (planes, programas y servicios) distan de responder a las necesidades. Aún subsiste el modelo hospitalario de tipo asilar que captura buena proporción de los presupuestos de salud mental, mientras que la participación de la atención primaria en la oferta de servicios de salud mental es limitada. Por otro lado, los trabajadores de la atención primaria están insuficientemente preparados para manejar de manera eficaz los problemas psicosociales (2). La Organización Panamericana de la Salud (OPS/ OMS), ha incrementado su apoyo técnico a los procesos de reforma en la región, lo cual se ha evidenciado en la Declaración de Caracas (1990) (4) y las Resoluciones del Consejo del Directivo de OPS/OMS (en los años 1997 y 2001) (5). Por medio de éstas se ha estimulado a los Estados Miembros a que:

- Desarrollen programas nacionales de salud mental.

- Reorienten los servicios de salud mental (de manicomiales a comunitarios).

- Desarrollen acciones de control de los trastornos afectivos, las epilepsias y las psicosis, como problemas prioritarios.

- Fortalezcan las acciones de promoción de la salud mental y el desarrollo psicosocial de la niñez.

- Aumenten las asignaciones para programas de formación en salud mental.

- Modernicen la legislación y protección de los derechos humanos de las personas con trastornos mentales y sus familiares.

Los procesos de reforma en salud mental requieren de información veraz y precisa; aunque el Informe Atlas de Salud Mental (OMS/2005) (6) provee de ciertos datos básicos, éstos no bastan para una adecuada planificación. En este artículo se presenta un grupo de indicadores sobre el estado de los sistemas de salud mental en El Salvador, Guatemala y Nicaragua; los mismos están basados en una evaluación que se llevó a cabo usando el Instrumento de Evaluación de los Sistemas de Salud Mental de la Organización Mundial de la Salud (WHO-AIMS, por sus siglas en inglés). Los resultados deben contribuir a promover los cambios y servir como una línea basal para monitorear el proceso.

\section{MÉTODO Y PROCEDIMIENTOS}

\section{El Instrumento de Evaluación para los Sistemas de Salud Mental}

El equipo de Evidencias e Investigación del Departamento de Salud Mental y Abuso de Sustancias de la OMS, conjuntamente con un grupo de consultores, desarrollaron el Instrumento de Evaluación para Sistemas de Salud Mental (WHO-AIMS) de la Organización Mundial de la Salud (OMS) (7), cuyo propósito es facilitar la recopilación de la información esencial sobre el sistema de salud mental de un país o región. Para el WHO-AIMS, un sistema de salud mental se define como todas aquellas actividades cuyos fines principales son promover, restaurar o mantener la salud mental, e incluye a todas las organizaciones y recursos orientados a lograr esos objetivos.

Las 10 recomendaciones destinadas a promover el mejoramiento de los servicios, publicadas en el Informe sobre la Salud en el Mundo-2001 (8), han servido como base para diseñar el WHOAIMS. Este instrumento permite evaluar los componentes clave e identificar las principales debilidades en los sistemas de salud mental, de tal manera que se obtiene información esencial para una acción pública relevante.
El WHO-AIMS consta de seis secciones que contienen 28 apartados y 154 ítems. Las secciones son interdependientes; están conceptualmente relacionadas y, hasta cierto punto, superpuestas; todas deben ser evaluadas a fin de tener una visión relativamente completa de un sistema de salud mental.

Al inicio de cada sección hay una lista de fuentes de datos recomendadas, las cuales son ajustadas de acuerdo con las características de los países. En algunos casos pueden existir otras fuentes relevantes de datos que no han sido enumeradas. En conjunto, se usan todas las fuentes de datos pertinentes y disponibles en el ámbito nacional, a nivel provincial/estatal/de distrito y al nivel de base (centro, unidad, servicio o dispositivo).

Los hallazgos del WHO-AIMS sirven para abogar por la introducción de reformas en salud mental, desarrollar planes y programas sustentados en parámetros iniciales claros y objetivos, monitorear el cambio y evaluar el progreso de las políticas de reforma, incluyendo la provisión de servicios comunitarios y la implicación de los usuarios, familias y otras partes interesadas en las acciones en salud mental.

\section{Recolección de la información}

El WHO-AIMS (7) se usó para recopilar información en El Salvador, Guatemala y Nicaragua. El trabajo se inició en mayo de 2005 con un taller celebrado en Managua, Nicaragua, donde se presentó el instrumento y se discutió su aplicación. Los respectivos ministerios de salud dieron su anuencia para efectuar la exploración y comprometieron su apoyo.

El siguiente paso fue capacitar a un equipo técnico de cada país en la aplicación del WHO-AIMS. Los equipos estuvieron integrados por un consultor nacional contratado por la OPS/OMS, el coordinador nacional de salud mental del Ministerio de Salud y el consultor de la Representación de OPS/OMS a cargo de salud mental. Además, cada país incorporó otros especialistas o funcionarios, según lo considerara conveniente. 
El consultor encargado de la recopilación de información y ejecución del WHO-AIMS contó con tres meses para realizar su trabajo; a lo largo del mismo mantuvo contactos sistemáticos con el equipo técnico que monitoreó y orientó el proceso en los tres países.

Al concluir la etapa de recolección de datos se convocó un taller nacional, con la participación de funcionarios y profesionales destacados provenientes del sector público y otras organizaciones, para la discusión y validación de los resultados. Las consideraciones más importantes fueron incorporadas al documento resumen de la situación diagnóstica. En octubre de 2005 se efectuó en San Salvador, El Salvador, un taller con asistencia de los tres países, así como del Asesor Subregional de Salud Mental de la OPS y un miembro del equipo de la OMS en Ginebra. En esta actividad se presentaron los informes respectivos, los cuales fueron objeto de una nueva discusión.

El equipo del WHO-AIMS en la sede de la OMS de Ginebra revisó todos los datos disponibles, y se hicieron aclaraciones y sugerencias que aseguraran una adecuada calidad de la información. El trabajo de campo se llevó a cabo en 2005 y los datos corresponden a 2004. La información obtenida proviene del sector público, integrado por los ministerios de salud e instituciones del seguro social en los países. La información del sector privado no pudo ser recolectada.

\section{Características generales de los países}

El Salvador, Guatemala y Nicaragua son países contiguos ubicados en el Istmo Centroamericano. Los tres comparten elementos comunes en su historia y en sus características económicas y sociales. A inicios del siglo XIX, cuando obtuvieron la independencia de España, formaban parte de la Federación Centroamericana, la cual posteriormente se disolvió. No obstante, los deseos de unidad han persistido a lo largo de casi 200 años; actualmente existen diferentes estructuras políticas dentro de marcos formales de integración centroamericana.

El Salvador. Cuenta con un área geográfica de 20646 kilómetros cuadrados y una población estimada de 6,5 millones de habitantes. El idioma oficial es el español. Los mestizos (mezcla de blancos e indígenas de origen maya) constituyen el principal grupo étnico. Las religiones predominantes son la católica y otras denominaciones cristianas. De los habitantes, 34\% tienen menos de 15 años y $8 \%$ más de 60 años. Por otra parte, $40 \%$ de la población es rural. La expectativa de vida al nacer es de 66,5 años para los hombres y de 72,8 para las mujeres. La tasa de alfabetización es de $82,4 \%$ para los varones y de 77,2 \% para las mujeres. La proporción del presupuesto de salud en el PIB es de 3,5. El país pertenece al grupo de ingresos medio-bajo, de acuerdo con los criterios de 2004 del Banco Mundial.

Guatemala. Posee una extensión territorial de 108889 kilómetros cuadrados, con una población de 12,7 millones de habitantes. Es un país pluricultural, multiétnico y multilingüístico, donde se identifican los siguientes grupos étnicos: blancos, ladinos (mestizos), mayas, xinkas y garífunas. El idioma oficial es el español, pero coexisten 21 lenguas mayas entre las que predominan el K'iche, el Mam, el Kaqchikel y el Q'ueqchí, además de los idiomas Xinka y Garífuna. Los grupos religiosos incluyen católicos, evangélicos y mayas. De la población, $43 \%$ reside en el área rural y $42 \%$ es indígena. Por otra parte, $62,6 \%$ es menor de 24 años y $18 \%$ corresponde a población adolescente; los mayores de 60 años representan $4 \%$ de la población. La expectativa de vida es de 65,9 años. En 2003, el gasto público en salud como porcentaje del PIB fue de 2,1. Esta nación pertenece al grupo de ingresos medio-bajo, de acuerdo con los criterios de 2004 del Banco Mundial.

Nicaragua. Su extensión geográfica es de 130682 kilómetros cuadrados y tiene 5,6 millones de habitantes. Se divide, además, en tres regiones geo- gráficas: Pacífico, Atlántico y Central, aunque los habitantes no están distribuidos homogéneamente sino que se concentran en la región del Pacífico que ocupa $15,3 \%$ del territorio nacional y alberga a $61,5 \%$ de la población total. El idioma oficial es el español pero, además, en la costa atlántica se habla el inglés y el dialecto miskito. Los principales grupos étnicos son mestizos, indígenas, negros y blancos. Los grupos religiosos más importantes incluyen católicos y diversas denominaciones cristianas. De la población, $41,2 \%$ tiene menos de 15 años y 4,7\% más de 60 años; $41 \%$ de la población es rural. La expectativa de vida al nacer es de 68,7 años para los hombres y 69,1 para las mujeres. La tasa de alfabetización es de 64\%. El Índice de Desarrollo Humano es el segundo más bajo de la región centroamericana. Nicaragua pertenece al grupo de ingreso bajo, según los criterios de 2004 del Banco Mundial. La proporción del presupuesto de salud en el PIB es de 3\%.

\section{Resultados de la evaluación}

\section{Politica y marco legislativo}

Política y planes. En ninguno de los tres países evaluados existen políticas nacionales de salud mental explícitas. Sin embargo, se han diseñado e implementado planes nacionales de salud mental, mismos que en el caso de Nicaragua y El Salvador fueron revisados y actualizados recientemente (2004-2005), mientras que el plan de Guatemala no ha sido objeto de revisión desde 1997. En 2004, con el apoyo técnico de la Organización Panamericana de la Salud (OPS/OMS), en los tres países se diseñaron planes de protección de la salud mental en situaciones de desastre y emergencias.

Legislación. Ninguna de las tres naciones cuenta con una legislación en materia de salud mental. No obstante, existen algunos elementos dispersos en la legislación vigente (Constitución de la República, Código o Ley de Salud, Código Penal, regulaciones sobre alcohol y tabaco, leyes sobre violencia y 
discapacidad, etc.), los cuales no alcanzan a constituirse en un cuerpo legal coherente de acuerdo con los modernos estándares internacionales.

Financiamiento de los servicios de salud mental. El comportamiento es similar en los tres países y distante de las recomendaciones de la OMS: "dada la carga de los trastornos mentales y neurológicos, la proporción del presupuesto general de salud destinado a la salud mental debería estar entre el 5 y 15\%" (7). En estas naciones sólo se dedica alrededor de $1 \%$ del presupuesto general de salud a la salud mental y, de éste, aproximadamente $90 \%$ se destina a los hospitales psiquiátricos. Es decir, los manicomios continúan absorbiendo la gran mayoría de los recursos financieros destinados a la salud mental, en detrimento de los servicios especializados ambulatorios y de atención primaria.

Para las personas pobres o con bajos niveles de ingreso, que tienen que pagar los medicamentos psicotrópicos, éstos resultan prácticamente inaccesibles. Así, la proporción del salario mínimo diario que dedicaría un usuario a la compra de la dosis diaria necesaria de un antipsicótico y de un antidepresivo en El Salvador, correspondería a $46 \%$ y $28 \%$, respectivamente; en Guatemala sería de $29 \%$ y $17 \%$, y en Nicaragua de $5 \%$ y $4 \%$.

Políticas sobre derechos humanos. En los tres países existen instituciones estatales dedicadas a la vigilancia y protección de los derechos humanos (Procuraduría de los Derechos Humanos), que si bien pueden realizar inspecciones o auditorías y atender quejas o reclamos, carecen de autoridad administrativa para aplicar sanciones, cerrar instituciones, etcétera. Sus recomendaciones no son vinculantes sino que, en la mayoría de los casos, sólo implican una sanción moral.

Cabe destacar que no existe una política de revisiones periódicas del estado de salvaguarda de los derechos humanos en los servicios hospitalarios de psiquiatría, como tampoco hay una capacitación sistemática para los trabajadores de los dispositivos de salud

CUADRO 1. Establecimientos de salud mental en El Salvador, Guatemala y Nicaragua, 2004

\begin{tabular}{|c|c|c|c|c|c|c|c|}
\hline País & $\begin{array}{c}\text { Servicio } \\
\text { ambulatorio }\end{array}$ & $\begin{array}{l}\text { Centro } \\
\text { de día }\end{array}$ & $\begin{array}{c}\text { Servicio de } \\
\text { psiquiatría } \\
\text { Hospital } \\
\text { General }\end{array}$ & $\begin{array}{c}\text { Hospital } \\
\text { psiquiátrico }\end{array}$ & $\begin{array}{l}\text { Establecimiento } \\
\text { residencial }\end{array}$ & $\begin{array}{l}\text { Unidad } \\
\text { forense }\end{array}$ & Total \\
\hline El Salvador & 49 & 0 & 0 & 2 & 9 & 1 & 52 \\
\hline Guatemala & 32 & 2 & 2 & 2 & - & 1 & 39 \\
\hline Nicaragua & 34 & 5 & 3 & 1 & 31 & 0 & 43 \\
\hline
\end{tabular}

mental. En 1998, el Grupo de trabajo para el diagnóstico sobre el estado de los derechos humanos de las personas con enfermedades mentales en países de Centroamérica, de la OPS/OMS, llevó a cabo una revisión del tema (9), y evaluaciones posteriores han hecho evidente el escaso avance logrado desde esa fecha hasta ahora.

\section{Servicios de salud mental}

A continuación se describe la situación de los diferentes tipos de servicios de salud mental en los países evaluados. En el cuadro 1 se detalla el número de dispositivos según categorías.

\section{Establecimientos de salud mental disponibles}

Servicios ambulatorios. La situación que priva en los tres países en cuanto a la existencia de servicios ambulatorios especializados dista de ser adecuada; esto es resultado de la distribución presupuestaria que continúa dando prioridad a los servicios hospitalarios. No obstante esta generalidad, se observan diferencias entre las naciones.

El Salvador cuenta con el mayor número de servicios ambulatorios y, en los últimos años, se han abierto algunos Centros Polivalentes de Atención Psicosocial que constituyen una experiencia novedosa en relación con la extensión de cobertura y búsqueda de alternativas al hospital psiquiátrico. Ejemplo importante en este contexto lo constituye la experiencia del Distrito Italia (Ilopango Norte), donde se desarrolla la integra- ción del servicio especializado con el Sistema Básico de Salud.

En Nicaragua han surgido los Centros de Atención Psicosocial (CAPs), que constituyen un modelo local de servicios comunitarios de salud mental. Su creación se remonta a la década de los años ochenta; empero, durante los noventa sufrieron un serio deterioro. Actualmente se están fortaleciendo algunos, como el de Chinandega que ha recibido el apoyo de la OPS/OMS mediante el proyecto WHO-AIMS.

La situación en Guatemala es crítica, pues escasean los establecimientos para una población relativamente numerosa. La mayoría de esos dispositivos apenas cuentan con un psicólogo. Cabe destacar que en este país, desde 1997, el Ministerio de Salud, con el apoyo de la Organización Panamericana de la Salud y otras agencias, ha desarrollado acciones de atención psicosocial a poblaciones indígenas que fueron afectadas por el conflicto armado. Recientemente, como parte del proyecto WHO-AIMS, se abrieron dos servicios ambulatorios de salud mental en las zonas 18 y 19 de la periferia de la ciudad capital. El valor agregado de estos servicios radica en que constituyen la fase inicial de la descentralización de la consulta externa del hospital.

Con respecto a los centros de día, El Salvador no cuenta con ninguno, Guatemala tiene dos (en los hospitales psiquiátricos de la capital) y Nicaragua cinco.

Existen muy pocos servicios o unidades de salud mental en hospitales generales. El desarrollo de esta modalidad asistencial es aún muy limitado e incipiente en los tres países. Se trata, 
en la mayoría de los casos, de unidades muy pequeñas, con pocos recursos, sin gran capacidad de respuesta y limitada cobertura. En El Salvador no existen; Guatemala cuenta con dos y Nicaragua con tres.

Los hospitales psiquiátricos están ubicados en las capitales de estos países y concentran la casi totalidad de las camas psiquiátricas. Continúan, en gran medida, con modelos de atención de tipo asilar, pobres condiciones de vida de las personas internadas y problemas con la protección de los derechos humanos. Con frecuencia, el promedio de estadía de internamiento sigue siendo alto; por ejemplo, más de $40 \%$ de los pacientes internados en el Hospital Psiquiátrico de Managua han estado allí por más de 10 años. Estos hospitales, generalmente, no responden a las necesidades crecientes de salud mental de la población.

Los establecimientos residenciales comunitarios (o casas de medio camino) destinados a enfermos con trastornos mentales de larga evolución, son casi inexistentes. Los que hay, son básicamente centros de tipo asilar, del sector privado, dedicados a ancianos o a casos con retraso mental y abuso de sustancias psicoactivas.

Las unidades psiquiátricas forenses se han desarrollado en los últimos años como una respuesta emergente a las demandas existentes en este campo. Nicaragua aún no dispone de un servicio de este tipo.

Atención en los servicios ambulatorios. El número de contactos por usuario varía desde 2,3 en El Salvador hasta 5,0 en Nicaragua. Guatemala no pudo notificar esta información. Debe señalarse, sin embargo, que éste es un dato obtenido mediante un mejor estimado o la evaluación de una muestra. Generalmente los sistemas registran de forma regular los contactos, pero no identifican el número de usuarios. La razón de usuarios atendidos por 100000 habitantes es de 782 en Guatemala, 627 en El Salvador y 145 en Nicaragua (cuadro 2).

Establecimientos ambulatorios dedicados exclusivamente a la infancia y

CUADRO 2. Establecimientos ambulatorios usuarios y contactos, El Salvador, Guatemala y Nicaragua, 2004

\begin{tabular}{lrrrr}
\hline \multicolumn{1}{c}{ País } & Usuarios & Contactos & $\begin{array}{c}\text { Contactos } \\
\text { por } \\
\text { usuario }\end{array}$ & $\begin{array}{c}\text { Usuarios } \\
\text { por } 100000 \\
\text { habitantes }\end{array}$ \\
\hline El Salvador & 42387 & 99113 & 2,34 & 627 \\
Guatemala & 99261 & - & - & 782 \\
Nicaragua & 8162 & 40812 & 5,0 & 145 \\
\hline
\end{tabular}

adolescencia. El número de servicios ambulatorios dedicados exclusivamente a la atención de salud mental de niños, niñas y adolescentes, es sólo una pequeña fracción de lo que realmente se necesita. Cabe pensar que una de las deudas pendientes en estos países es la necesidad de incrementar y mejorar la atención infanto-juvenil en salud mental, dado que en El Salvador sólo $2 \%$ de sus servicios ambulatorios se dedican exclusivamente a la infancia y la adolescencia (1/49); en Guatemala esa proporción es de 3\% $(1 / 32)$ y en Nicaragua de $8 \%(3 / 34)$.

Tipo de oferta disponible en los servicios ambulatorios y calidad de la atención:

- Guatemala y Nicaragua carecen de psicofármacos en los servicios ambulatorios, en tanto que en El Salvador sólo $49 \%$ de los mismos tienen medicamentos psicotrópicos.

- Se realizan intervenciones de tipo psicosocial exclusivamente en algunos servicios ambulatorios (entre 21 y 50\%), según un mejor estimado.

- El seguimiento de casos en la comunidad es limitado o inexistente (ningún servicio en Guatemala, 6\% en El Salvador y $50 \%$ en Nicaragua).

- No existen equipos móviles de salud mental.

\section{Hospitales psiquiátricos}

Los tres principales diagnósticos en las personas internadas en hospitales psiquiátricos son: esquizofrenias, trastornos afectivos y trastornos asociados al consumo de sustancias psicoactivas.
En El Salvador, los trastornos asociados al consumo de sustancias son los más frecuentes, en lo cual puede influir la existencia de un importante servicio de adicciones dentro del hospital.

La reducción de camas en los hospitales psiquiátricos en los últimos cinco años ha sido poco significativa: $7 \%$ en El Salvador y ninguna en Nicaragua y Guatemala.

La admisión involuntaria de casos y la restricción física o aislamiento de casos no se controla ni registra de manera sistemática. Sólo en El Salvador se hizo un estimado al respecto mediante revisión de expedientes clínicos, y se encontró que $35 \%$ de las admisiones fueron involuntarias $y$ alrededor de $8 \%$ de los casos internados en los hospitales psiquiátricos fueron sometidos a medidas de restricción física o aislados (cuadro 3).

\section{Equidad en el acceso a los servicios de salud mental}

Los tres países informaron índices evidentes de inequidad, como los siguientes:

- Las camas psiquiátricas están concentradas, casi en su totalidad, en las capitales, de tal manera que en El Salvador la relación entre la razón de camas psiquiátricas de todo el país y las que se encuentran en la capital, es de 2,90, mientras que en Guatemala es de 3,34 y en Nicaragua de 5,39.

- Los usuarios de zonas rurales, los grupos indígenas y otras minorías étnicas y lingüísticas, están signifi- 
CUADRO 3. Hospitales psiquiátricos; datos seleccionados de El Salvador, Guatemala y Nicaragua, 2004

\begin{tabular}{|c|c|c|c|c|c|c|}
\hline \multirow[b]{2}{*}{ País } & \multicolumn{2}{|c|}{$\begin{array}{l}\text { Camas en hospitales } \\
\text { psiquiátricos }\end{array}$} & \multirow{2}{*}{$\begin{array}{c}\text { Promedio } \\
\text { de estadía } \\
\text { (días) }\end{array}$} & \multirow{2}{*}{$\begin{array}{c}\text { Índice } \\
\text { ocupacional } \\
(\%)\end{array}$} & \multirow{2}{*}{$\begin{array}{l}\text { Reducción } \\
\text { de camas } \\
\text { en } 5 \text { años }\end{array}$} & \multirow{2}{*}{$\begin{array}{c}\text { Diagnósticos } \\
\text { más } \\
\text { frecuentes }\end{array}$} \\
\hline & No. & Razóna & & & & \\
\hline El Salvador & 426 & 6,30 & 16,8 & 49 & $7 \%$ & $\begin{array}{l}\text { 1. Trastornos asociados } \\
\text { al consumo de } \\
\text { alcohol y drogas } \\
\text { 2. Trastornos afectivos } \\
\text { 3. Esquizofrenia }\end{array}$ \\
\hline Guatemala & 336 & 2,65 & 75 & 85 & - & $\begin{array}{l}\text { 1. Esquizofrenia } \\
\text { 2. Trastornos afectivos } \\
\text { 3. Trastornos asociados } \\
\text { al consumo de } \\
\text { alcohol y drogas }\end{array}$ \\
\hline Nicaragua & 168 & 2,99 & 69,9 & 122 & - & $\begin{array}{l}\text { 1. Esquizofrenia } \\
\text { 2. Trastornos afectivos } \\
\text { 3. Trastornos asociados } \\
\text { al consumo de } \\
\text { alcohol y drogas }\end{array}$ \\
\hline
\end{tabular}

aPor 100000 habitantes.

cativamente subrepresentados en el uso de los servicios ambulatorios de salud mental.

- No existen estrategias formales que garanticen el acceso equitativo a los servicios de salud mental para los grupos indígenas y otras minorías étnicas y lingüísticas.

\section{Salud mental en la atención primaria de salud}

Para los efectos de este análisis, se evaluaron los programas educativos de formación de médicos y enfermeras a fin de determinar el tiempo dedicado a los temas de salud mental en la formación de pregrado (universitaria). Para el estudio se incluyeron todas las universidades de cada país (públicas o privadas) donde se impartían las carreras de medicina y enfermería. Se destaca que, en Guatemala, tanto la asignatura de psiquiatría como otros contenidos de salud mental desaparecieron, casi totalmente, del programa de estudios de la facultad de medicina de la principal universidad pública del país, de tal manera que se les dedica tan sólo 1\% del tiempo de formación de los médicos, y 3\% del correspondiente a las enfermeras; en El Salvador se destina $7 \%$ en el caso de los médicos y $4 \%$ para las enfermeras $y$, final- mente, en Nicaragua las proporciones son $2 \%$ y $7 \%$, respectivamente.

En las tres naciones, los programas de capacitación y educación continua de post-grado son impartidos, fundamentalmente, por los ministerios de salud y, en menor medida, por las instituciones de seguridad social. El primer elemento que cabe destacar es que los datos no estaban disponibles en los sistemas regulares de información; por lo tanto, fue necesario realizar un mejor estimado mediante la recopilación en diversas fuentes. En Guatemala no fue posible contar con la información.

En sentido general, se evidencia la casi inexistencia de programas regulares de capacitación en temas de salud mental para el personal de atención primaria de salud (APS). Los pocos resultados que se muestran son producto de cursos aislados o procesos no sistemáticos. En El Salvador $16 \%$ de los médicos de APS, y 13\% de las enfermeras, fueron capacitados en salud mental; en Nicaragua ninguna enfermera y sólo $4 \%$ de los médicos de APS recibieron esa capacitación. En Guatemala no se disponía de información al respecto.

El Salvador es el país que más ha trabajado en el desarrollo de normas y protocolos de atención en salud mental dirigidos a trabajadores de APS; éstos se encuentran validados y se ha comenzado su implementación en algunos centros de APS (menos de 20\%). Guatemala dispone de protocolos elaborados a finales de los años noventa que no han sido revisados ni actualizados; se encuentran actualmente implementados en menos de $20 \%$ de los centros de APS. En Nicaragua no se dispone de protocolos.

La disponibilidad de psicofármacos en la APS es crítica en Nicaragua y Guatemala, ya que no están disponibles estos medicamentos. En El Salvador hay medicamentos disponibles en un grupo de centros que se estima entre $21 \%$ y $50 \%$.

Las modalidades de prescripción de los psicofármacos son similares en los tres países: pueden prescribir sin limitaciones (o con muy pocas) los psiquiatras y otros médicos de la APS. Está prohibido que las enfermeras u otro tipo de personal no médico prescriba medicamentos.

\section{Recursos humanos}

Para evaluar la disponibilidad de recursos humanos calificados en los establecimientos de salud mental (todos los tipos), se seleccionaron tres categorías de trabajadores; en el cuadro 4 se muestran las cifras numéricas y la razón por 100000 habitantes.

Los psiquiatras son un integrante fundamental de los equipos de salud mental; si son insuficientes, la atención de salud mental se afecta o limita sensiblemente El indicador de un psiquiatra o menos por cien mil habitantes coloca a estos países en el grupo de naciones con el más bajo número de psiquiatras por población, la razón.

Por otra parte, la elevada concentración de los recursos humanos en las capitales afecta seriamente el acceso a los servicios de la población del interior de la nación, en especial de las zonas rurales o de los indígenas. En Guatemala, la relación entre la razón nacional de médicos psiquiatras y la de aquellos ubicados en la capital es de 2,96; en El Salvador es de 2,59 y en Nicaragua de 3,48 . 
CUADRO 4. Recursos humanos en establecimientos de salud mental en El Salvador, Guatemala y Nicaragua, 2004

\begin{tabular}{|c|c|c|c|c|c|c|}
\hline \multirow[b]{2}{*}{ Recursos humanos } & \multicolumn{2}{|c|}{ El Salvador } & \multicolumn{2}{|c|}{ Guatemala } & \multicolumn{2}{|c|}{ Nicaragua } \\
\hline & No. & Razón ${ }^{a}$ & No. & Razóna & No. & Razóna \\
\hline Médicos psiquiatras & 94 & 1,39 & 73 & 0,57 & 51 & 0,91 \\
\hline Enfermeras & 143 & 2,12 & 162 & 1,28 & 96 & 1,71 \\
\hline Psicólogos & 114 & 1,69 & 44 & 0,35 & 119 & 2,11 \\
\hline
\end{tabular}

a Por 1000000 habitantes.

CUADRO 5. Disponibilidad de profesionales de salud mental y actividades de promoción de la salud mental y prevención de los trastornos psíquicos en escuelas primarias y secundarias de El Salvador, Guatemala y Nicaragua, 2004

\begin{tabular}{|c|c|c|c|}
\hline \multirow[b]{2}{*}{ País } & \multicolumn{2}{|c|}{$\begin{array}{c}\text { Escuelas con } \\
\text { disponibilidad de } \\
\text { profesionales mental }\end{array}$} & \multirow{2}{*}{$\begin{array}{l}\text { Escuelas donde se realizan } \\
\text { actividades de salud } \\
\text { de promoción de la salud } \\
\text { mental y prevención } \\
\text { de los trastornos psíquicos }\end{array}$} \\
\hline & No. & $\%$ & \\
\hline El Salvador & 31 & 1 & $51-80 \%$ \\
\hline Guatemala & 41 & 2 & $<20 \%$ \\
\hline Nicaragua & 829 & 12 & $<20 \%$ \\
\hline
\end{tabular}

\section{Educación del público y vínculos con otros sectores}

Actividades de promoción de la salud mental y prevención en escuelas. En sentido general, se observa que en los sistemas públicos de educación es muy bajo el número de escuelas que pueden contar con un profesional de salud mental. Sin embargo algunos países, como El Salvador, han logrado capacitar a maestros y trabajadores de APS para que desarrollen acciones de promoción y prevención en las escuelas (entre $51 \%$ y $80 \%$, según el mejor estimado). El porcentaje es mucho menor en Guatemala y Nicaragua (menos de 20\%) (cuadro 5).

Asociaciones de usuarios y familiares. En general, la presencia y el grado de desarrollo de las asociaciones de usuarios y familiares son muy limitados en las tres naciones. La membresía es pobre numéricamente $y$, casi siempre, mixta (usuarios y familiares). No reciben subsidios ni aportes económicos de los gobiernos y casi nunca participan de manera significativa en los procesos de planificación e implemen- tación de políticas, programas y legislación. Su interacción con los servicios de salud mental es muy limitada.

\section{Evaluación e investigación}

Los sistemas de información adolecen de múltiples deficiencias:

- No todas las unidades o servicios de salud mental informan a los ministerios de salud.

- Los datos que se recolectan son insuficientes para un análisis adecuado.

- Existe subregistro o mala calidad en el registro primario.

- No se dispone de datos del sector privado.

- No hay publicaciones o informes anuales sobre salud mental.

- No existen sistemas de vigilancia epidemiológica.

La falta de investigación es, sin lugar a dudas, una debilidad de los países evaluados. En El Salvador 12\% de las investigaciones notificadas formalmente (PubMed) correspondieron a salud mental; en Nicaragua ascendieron a $24 \%$, y en Guatemala no se informó de ninguna. La opinión generalizada es que no se dispone de recursos para esta área que, además, no es prioritaria para el sector público. Una excepción es Nicaragua, ya que ha tenido un cierto grado de desarrollo con un equipo de investigadores en salud mental en la Universidad de León.

\section{Recomendaciones para la acción}

Existen tres documentos adicionales que pueden ayudar a los países como guía para la reforma de los servicios de salud mental: las recomendaciones del Informe Mundial de Salud (OMS, 2001), las resoluciones de la OPS/OMS y la declaración final de Brasilia (OPS, 2005).

Las 10 recomendaciones del Informe sobre la Salud en el Mundo (7) pueden utilizarse como guía de acción; éstas, también, fueron el fundamento para el diseño del instrumento de evaluación de los sistemas de salud (WHO-AIMS):

1. Dispensar tratamiento en la APS.

2. Garantizar la disponibilidad de medicamentos psicotrópicos.

3. Prestar asistencia en la comunidad.

4. Educar al público.

5. Involucrar a las comunidades, las familias y los consumidores.

6. Establecer políticas, programas y legislación a escala nacional.

7. Desarrollar los recursos humanos.

8. Establecer vínculos con otros sectores.

9. Vigilar la salud mental de las comunidades.

10. Apoyar nuevas investigaciones.

De otro lado, en noviembre de 2006 la Conferencia Regional para la Reforma de los Servicios de Salud Mental: 15 años después de Caracas, efectuada en Brasilia, aprobó los "Principios rectores para el desarrollo de la atención de salud mental en las Américas". En esa conferencia se reiteró la vigencia de los elementos fundamentales de la Declaración de Caracas (1990) y se advirtió que los servicios de salud deben afrontar nuevos desafíos técnicos y cul- 
turales. La declaración final constituye otro elemento de guía para el trabajo.

A continuación se mencionan algunas recomendaciones para la acción en el corto, mediano y largo plazo para los tres países.

En el corto plazo:

1. Formular una política nacional de salud mental que sirva de marco estratégico a los planes operativos y focalice las prioridades.

2. Incluir la salud mental en programas de acceso a medicamentos para la población de bajos ingresos. Es necesario dar prioridad a la existencia de medicamentos gratuitos o subsidiados en los centros de APS y servicios de salud mental ambulatorios.

3. Incrementar y fortalecer los modelos de servicios comunitarios de salud mental, asignándoles los recursos humanos y financieros adecuados. Como ilustración se mencionan experiencias positivas que se han desarrollado o fortalecido mediante el Proyecto WHO-AIMS:

a. Centros de Atención Psicosocial en Nicaragua.

a. Centros Polivalentes de Atención Psicosocial en El Salvador.

c. Unidades de Salud Mental en las Clínicas Periféricas de la Ciudad de Guatemala.

4. Movilizar o cambiar algunos recursos de los hospitales hacia los servicios de base comunitaria.

5. Desarrollar la atención de salud mental a la infancia y adolescencia. Se recomienda implementar un programa específico adecuado a las condiciones y recursos de cada país.

6. Diseñar e implementar un programa regular de capacitación para trabajadores de la APS, que garantice que en dos años se puede capacitar, al menos, a $80 \%$ de este personal.

7. Fortalecer los sistemas de información y vigilancia epidemiológica.

En el mediano y largo plazo:

\section{Política y marco legislativo}

1. Proponer a las instancias correspondientes las reformas necesarias para actualizar la legislación en el campo de la salud mental, de acuerdo con los estándares más modernos.

2. Incrementar el presupuesto dedicado a la salud mental, buscando en una primera etapa un mínimo de $3 \%$ del presupuesto general de salud (algunos países de Centroamérica, como Panamá, han alcanzado este objetivo) y cambiar la estructura del gasto dentro del sistema de salud mental, garantizando que los servicios comunitarios y ambulatorios tengan la prioridad por encima de los hospitales psiquiátricos.

3. Establecer convenios entre los ministerios de salud y las procuradurías de los derechos humanos, para regularizar la inspección y vigilancia de los derechos humanos de los enfermos mentales, asegurando la salvaguarda de los mismos.

\section{Servicios de salud mental}

4. Descentralizar la consulta externa de los hospitales psiquiátricos hacia servicios ambulatorios en las tres naciones.

5. Promover la apertura de servicios de psiquiatría en los hospitales generales más importantes de cada país, en especial los de carácter departamental y regional.

6. Desarrollar centros de día vinculados a los servicios ambulatorios de salud mental o los servicios de psiquiatría de hospitales generales.

7. Abrir una unidad forense en Nicaragua y fortalecer las existentes en Guatemala y El Salvador.

8. Diseñar e implementar estrategias y acciones específicas que permitan el acceso equitativo a los servicios a los usuarios de zonas rurales, así como a los indígenas y a otras minorías étnicas, culturales y lingüísticas.

\section{Salud mental en la atención primaria} de salud

9. Aprobar las normas y protocolos de atención en salud mental para la APS. El Salvador puede aportar su experiencia en este sentido. La meta debe ser su implementación en no menos de $80 \%$ de los centros.

\section{Recursos humanos}

10. Diseñar un programa de conjunto universidades-ministerio, para la formación y recalificación de los recursos humanos especializados en salud mental; establecer metas de corto, mediano y largo plazo.

11. Establecer estrategias para la asignación de los recursos humanos, garantizando la cobertura de servicios de salud mental en zonas rurales y del interior de los países.

Educación del público y vínculos con otros sectores

12. Establecer convenios entre los ministerios de educación y salud, para asegurar la designación progresiva de profesionales de salud mental en las escuelas públicas primarias y secundarias; alentar, en el mismo sentido, al sector privado. En paralelo, capacitar a maestros y trabajadores de APS para que, de manera conjunta, desarrollen acciones de promoción de la salud y prevención de los trastornos psíquicos en las escuelas.

13. Apoyar las organizaciones de usuarios y familiares, incentivando su participación activa en los planes y programas de salud mental y favoreciendo su interacción sistemática con los servicios.

\section{Evaluación e investigación}

14. Formar grupos de trabajo en cada país, con participación conjunta de las universidades y las instituciones públicas para impulsar la investigación. La OPS/OMS puede apoyar esta iniciativa y la Universidad de León en Nicaragua puede desempeñar un papel de liderazgo en este proceso.

\section{CONCLUSIONES}

Nicaragua, Guatemala y El Salvador tienen importantes limitaciones en sus sistemas nacionales de salud mental. Es clara la marcada insuficiencia de recursos humanos calificados. El presupuesto dedicado a la salud mental apenas representa $1 \%$ del presupuesto general de salud y los hospitales psi- 
quiátricos situados en la capital de los países consumen más de $90 \%$ de los fondos asignados a la salud mental.

Un problema clave es el pobre desarrollo del componente salud mental en la APS. Los hospitales psiquiátri-cos continúan siendo hegemónicos a pesar de algunos avances logrados en la asistencia ambulatoria y de la positiva concepción de trabajo desarrollada en los planes nacionales de salud mental.

La supervisión del respeto de los derechos humanos de los enfermos mentales debe fortalecerse. También se hace necesario sistematizar los procesos de capacitación y actualización de los profesionales de la salud mental y los trabajadores de la APS.

Se recomienda que los países definan un grupo de prioridades y se establezca un plan de acción de corto y mediano plazo que permita avanzar en aspectos básicos de desarrollo de los servicios. Como un elemento positivo se destaca que, en los últimos años, se ha logrado impulsar algunas experiencias innovadoras que requieren generalizarse.
Reconocimientos. La recopilación de información en Nicaragua, El Salvador y Guatemala estuvo a cargo de los equipos técnicos nacionales, con la participación de los respectivos ministerios de salud. El proyecto contó con el apoyo las Representaciones de la OPS/OMS en los países, así como de la Unidad Regional de Salud Mental y Programas Especializados de la Organización Panamericana de la Salud (OPS/OMS). Asimismo recibió el apoyo, desde la sede de la OMS en Ginebra, del Equipo de Evidencias e Investigación del Departamento de Salud Mental y Abuso de Sustancias. El equipo del WHO-AIMS incluye a: Benedetto Saraceno, Shekhar Saxena (quien lo coordina), Tom Barrett, Antonio Lora, Mark van Ommeren, Jodi Morris y Grazia Motturi. Los participantes directos en Centroamérica fueron: Coordinación y revisión técnica de los tres informes: Thomas Barrett, Asesor de la OMS, Ginebra; Jorge Rodríguez, Asesor en Salud Mental para Centroamérica, OPS/OMS; Silvia Narváez, OPS/OMS Nicaragua, y Carlos Ma- nuel Fernández, Consultor. Informe de Nicaragua: Carlos Manuel Fernández, Consultor Nacional; Carlos Fletes, Jefe del Programa Nacional de Salud Mental de Nicaragua, y la Silvia Narváez, OPS/OMS Nicaragua. Informe de Guatemala: José Antonio Flores, Consultor Nacional; Aura Marina López, Marline Paz y Nadyezhda van Tullen, del Programa Nacional de Salud Mental; Edgar R. Vásquez, médico psiquiatra, y José Adán Montes, OPS/OMS Guatemala. Informe de El Salvador: Arturo Carranza, Consultor Nacional; Roberto Rivas, Director de Regulación del Ministerio de Salud Pública y Asistencia Social de El Salvador; Moisés Guardado, Jefe del Programa Nacional de Salud Mental; Ulises Gutiérrez, Director del Hospital Psiquiátrico de San Salvador y Amalia E. Ayala, OPS/ OMS El Salvador. El Proyecto WHOAIMS está parcialmente financiado por el Instituto Nacional de Salud Mental y la Administración de Servicios de Salud Mental y Abuso de Sustancias de los Estados Unidos de América.

\section{REFERENCIAS}

1. Kohn R, Levav I, Donaire I, Machuca M, Tamashiro R. Psychological and psychopathological reactions in Honduras following Hurricane Mitch: implications for services planning. Rev Panam Salud Publica. 2005;18(4/5): 287-95.

2. Organización Panamericana de la Salud. Informe sobre los Sistemas de Salud Mental en Nicaragua, El Salvador y Guatemala. Documentación de OPS/OMS, 2006.

3. Rodríguez J, Bergonzoli G, Levav I. Violencia política y salud mental en Guatemala. Acta Psiquiatr Psicol Am Lat. 2002;48(1-4):43-9.

4. Levav I, González R, eds. Reestructuración de la atención psiquiátrica: bases conceptuales y guías para su implementación. Washing- ton D.C.: Organización Panamericana de la Salud - Instituto Mario Negri; 1991.

5. Organización Panamericana de la Salud/ Organización Mundial de la Salud. Salud Mental. Resolución CD43/ 15. 43 Consejo Directivo. Washington D.C., 24 al 28 de septiembre de 2001. Hallado en URL: http://www.paho. org/spanish/gov/cd/cd43_15-s.pdf Acceso el 24 de febrero 2007.

6. World Health Organization. Mental Health Atlas-2005. Geneva: World Health Organization; 2005. WHO.

7. World Health Organization. Assessment Instrument for Mental Health Systems. Version 2.2. Geneva: WHO Document Production Services; 2005.
8. World Health Organization. The World Health Report 2001. Mental Health: New Understanding, New Hope. Geneva: World Health Organization; 2001.

9. Organización Panamericana de la Salud. Grupo de trabajo para el diagnóstico sobre el estado de los derechos humanos de las personas con enfermedades mentales en países de Centroamérica. Informe final. Tegucigalpa: OPS/OMS; 1998.

Manuscrito recibido el 23 de febrero de 2007. Aceptado para publicación, tras revisión, el 25 de septiembre de 2007. 
ABSTRACT The authors evaluated the mental health systems of El Salvador, Guatemala, and Nicaragua, using a group of select indicators. The World Health Organization Assessment Instrument for Mental Health Systems (WHO-AIMS) was used to collect

Mental health systems in El Salvador, Guatemala, and Nicaragua: results of a WHO-AIMS evaluation data from the nations. The national mental health systems of Nicaragua, Guatemala, and El Salvador have serious limitations, especially in primary care, and a marked lack of qualified human resources. Budget allocations for mental health care are barely $1 \%$ of the total health care budgets; and the psychiatric hospitals located in the national capitals consume at least $90 \%$ of those funds. The limited human and material resources available are concentrated in the respective country capital cities. National mental health policies and legislation have not been adopted; however, all three countries do have national plans in progress. Furthermore, all three have designed and implemented programs for mental health care in case of disaster. Agreements must be reached with offices for the defense of human rights to raise awareness and protection of rights for the mentally ill.

In recent years, new experiences have been gained and these should be distributed more widely. The Pan American Health Organization (PAHO/WHO) and the WHOAIMS project have contributed toward developing community models for mental health services. Lastly, priorities have been identified and action items recommended

Key words Mental health; mental health services; health care quality, access, and evaluation; El Salvador, Guatemala, Nicaragua. 